\title{
THE SIGNLESS LAPLACIAN SEPARATOR OF GRAPHS*
}

\author{
ZHIFU YOU ${ }^{\dagger}$ AND BOLIAN $\mathrm{LIU}^{\dagger}$
}

\begin{abstract}
The signless Laplacian separator of a graph is defined as the difference between the largest eigenvalue and the second largest eigenvalue of the associated signless Laplacian matrix. In this paper, we determine the maximum signless Laplacian separators of unicyclic, bicyclic and tricyclic graphs with given order.
\end{abstract}

Key words. Signless Laplacian, Separator, $c$-cyclic graphs.

AMS subject classifications. 15A18, 05C50.

1. Introduction. Let $G$ be a simple graph with $n$ vertices and $m$ edges. A connected graph is called a $c$-cyclic graph if $c=m-n+1$. A connected graph is unicyclic (resp., bicyclic, tricyclic) when $m=n$ (resp., $m=n+1, m=n+2$ ). Denote by $\delta(G)$ and $\Delta(G)$ the minimum and the maximum degree, respectively. Let $A$ be the adjacency matrix of $G$. Since $A$ is symmetric, its eigenvalues are real and can be written in descending order: $\lambda_{1}(G) \geq \lambda_{2}(G) \geq \cdots \geq \lambda_{n}(G)$. The Laplacian matrix of $G$ is defined as $L=D-A$, where $D=\operatorname{diag}\left(d_{1}, d_{2}, \ldots, d_{n}\right)$ is the diagonal matrix of vertex degrees. The Laplacian spectrum of $G$ (the spectrum of its Laplacian matrix) consists of the values $\mu_{1}(G) \geq \mu_{2}(G) \geq \cdots \geq \mu_{n}(G)=0$. The signless Laplacian matrix of $G$ is defined as $Q=D+A$. Denote the eigenvalues of $Q$ by $q_{1}(G) \geq q_{2}(G) \geq \cdots \geq q_{n}(G) \geq 0$.

The separator $S_{A}(G)$ of $G$ is the difference between its largest and second largest eigenvalues, i.e., $S_{A}(G)=\lambda_{1}(G)-\lambda_{2}(G)$. In [1], Li et al. defined the Laplacian separator $S_{L}(G)$ as the difference between its largest and second largest Laplacian eigenvalues, i.e., $S_{L}(G)=\mu_{1}(G)-\mu_{2}(G)$.

The set of trees, unicyclic graphs and bicyclic graphs of order $n$ are denoted by $T_{n}, U_{n}$ and $B_{n}$, respectively.

Li et al. [1] obtained:

TheOrem 1.1. ([1]) If $T \in T_{n}$ with $n \geq 4$, then $S_{A}(T) \leq \sqrt{n-1}$. Equality holds

${ }^{*}$ Received by the editors on July 22, 2010. Accepted for publication on February 17, 2011. Handling Editor: Bryan Shader.

${ }^{\dagger}$ School of Mathematical Science, South China Normal University, Guangzhou, 510631, P.R. China (youzhf@hotmail.com, Zhifu You; liubl@scnu.edu.cn, Bolian Liu). This work was supported by the NNSF of China (No. 11071088). 
if and only if $T \cong K_{1, n-1}$.

THEOREM 1.2. ([1]) (1) If $T \in T_{n}$ with $n \geq 3$, then $S_{L}(T) \leq n-1$, and equality holds if and only if $T \cong K_{1, n-1}$.

(2) For any $T \in T_{n}$ with $n \geq 6$, if $T$ is neither $K_{1, n-1}$ nor $T_{n}^{2}$, then $S_{L}(T)<$ $n-\frac{5+\sqrt{5}}{2}$, where $T_{n}^{2}$ is the tree obtained by joining two isolated vertices to two pendant vertices of $K_{1, n-2}$.

THEOREM 1.3. ([1]) If $U \in U_{n}$ with $n \geq 6$, then $S_{L}(U) \leq \sqrt{n-3}$, and equality holds if and only if $U \cong U_{n}^{1}$, where $U_{n}^{1}$ is the graph obtained by attaching $n-3$ pendant vertices to a common vertex of $C_{3}$.

Similarly, the signless Laplacian separator $S_{Q}(G)$ is the difference between its largest and second largest signless Laplacian eigenvalues, i.e., $S_{Q}(G)=q_{1}(G)-q_{2}(G)$.

In fact, Das [2] has proved five conjectures among a series of 30 conjectures (see [3]) on Laplcian eigenvalues and signless Laplacian eigenvalues of $G$. And two conjectures are related to the signless Laplacian separator.

CONJECTURE 1.4. ([3]) If $G$ is a connected graph of order $n \geq 4$, then $q_{1}-q_{2} \leq n$ with equality holding if and only if $G \cong K_{n}$.

Conjecture 1.5. ([3]) If $T$ is a tree of order $n \geq 4$, then $q_{1}-q_{2} \leq n-1$ with equality holding if and only if $G \cong K_{1, n-1}$.

It is well known that the Laplacian matrix and signless Laplacian matrix of $G$ have the same eigenvalues when $G$ is bipartite (see [4]). Then Conjecture 1.5 can be directly deduced from Theorem 1.2.

In this paper, we determine the maximum signless Laplacian separators of unicyclic, bicyclic and tricyclic graphs with given order.

2. Preliminaries. We refer to the signless Laplacian eigenvalues as $Q$-eigenvalues.

In this section, we present some lemmas about $Q$-eigenvalues.

Lemma 2.1. ([5]) For $e \notin E(G)$, the $Q$-eigenvalues of $G$ and $G^{\prime}=G+e$ interlace, i.e.,

$$
q_{1}\left(G^{\prime}\right) \geq q_{1}(G) \geq q_{2}\left(G^{\prime}\right) \geq q_{2}(G) \geq \cdots \geq q_{n}\left(G^{\prime}\right) \geq q_{n}(G) .
$$

Denote by $d(v)$ and $N(v)$ the degree and set of neighbours of vertex $v$, respectively. 
LEMma 2.2. ([6]) $q_{1}(G) \leq \max \{d(v)+m(v): v \in V\}$ and $m(v)=\sum_{u \in N(v)} \frac{d(u)}{d(v)}$.

LEMma 2.3. ([7]) Suppose $c \geq 0$, and $G$ is a c-cyclic graph with $n$ vertices with $\Delta \leq n-2$. If $n \geq \max \{c+5,2 c+3\}$, then $q_{1}(G) \leq n$.

In [2], Das obtained:

LEMMA 2.4. ([2]) If $G$ is a connected graph, then $q_{1}-q_{2} \leq \Delta+1$.

We will determine the maximum $Q$-separator among unicyclic, bicyclic, and tricyclic graphs according to the maximum degree $\Delta$.

By Lemma 2.4, it is easy to see:

LEMMA 2.5. If $G$ is connected and $\Delta(G) \leq n-4$, then $S_{Q}(G) \leq n-3$.

In the following, we first characterize the subgraphs which are contained in a $c$-cyclic graphs with $\Delta=n-3$ or $\Delta=n-2$.

Lemma 2.6. Let $G$ be a connected $c$-cyclic graph with $n \geq 7$ vertices. If $c \geq 1$ and $\Delta(G)=n-3$, then $G$ must have one of $H_{i}(i=1,2, \ldots, 12)$ as a subgraph, where $H_{i}$ are depicted in Figure 1.

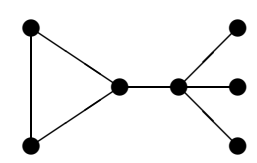

$H_{1}$

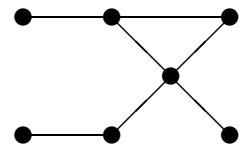

$H_{5}$

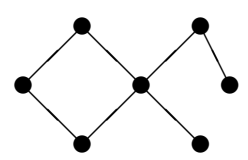

$H_{9}$

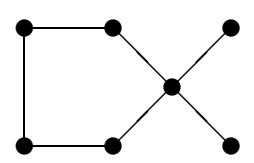

$\mathrm{H}_{2}$

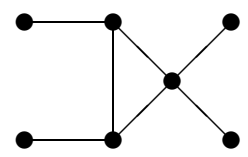

$H_{6}$

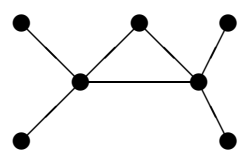

$H_{10}$

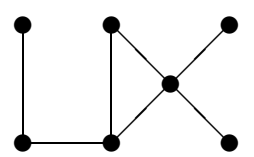

$H_{3}$

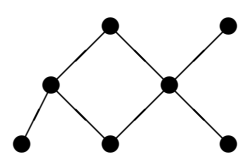

$\mathrm{H}_{7}$

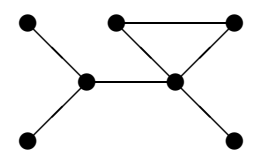

$H_{11}$

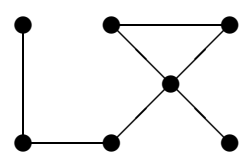

$\mathrm{H}_{4}$

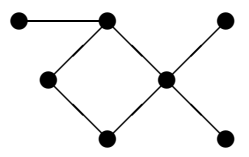

$H_{8}$

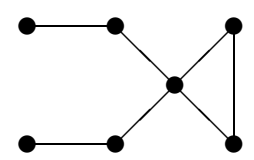

$H_{12}$

Figure 1. Subgraphs contained in $c$-cyclic graphs with $c \geq 1$ and $\Delta=n-3$.

Proof. Since $\Delta=n-3, G$ has the subgraph $K_{1, n-3}$ and two vertices non-adjacent to the $\Delta$-degree vertex. Note that $c \geq 1$. Then $G$ has one of $H_{1}, H_{2}, \ldots, H_{12}$ as a subgraph. $\square$ 
TABLE 2.1

The second largest $Q$-eigenvalues of $H_{i}(i=1,2, \ldots, 12)$.

\begin{tabular}{cccccccccc}
\hline$H_{1}$ & $H_{2}$ & $H_{3}$ & $H_{4}$ & $H_{5}$ & $H_{6}$ & $H_{7}$ & $H_{8}$ & $H_{9}$ & $H_{10}$ \\
\hline 4 & 3.3675 & 3.3880 & 3.2149 & 3.2470 & 3.2384 & 3.8360 & 3.4142 & 3.1129 & 3.7321 \\
\hline$H_{11}$ & $H_{12}$ & & & & & & & & \\
\hline 3.5565 & 2.7935 & & & & & & & & \\
\hline
\end{tabular}

Lemma 2.7. Let $G$ be a connected c-cyclic graph with $n \geq 7$ vertices. If $c \geq 1$ and $\Delta(G)=n-2$, then $G$ contains one of $H_{j}(j=13,14,15)$ as a subgraph, where $H_{j}$ are depicted in Figure 2.

Proof. Since $\Delta=n-2, G$ has the subgraph $K_{1, n-2}$ and one vertex non-adjacent to the $\Delta$-degree vertex. Note that $c \geq 1$. Then $G$ has one of $H_{13}, H_{14}, H_{15}$ as a subgraph.
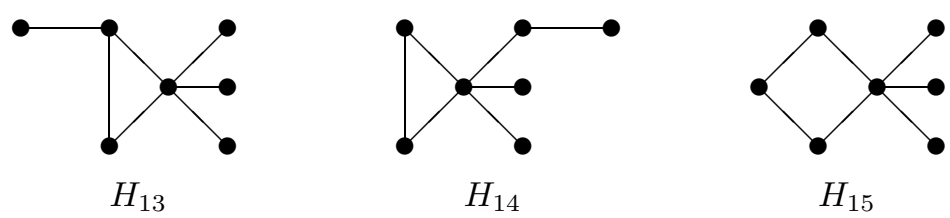

Figure 2. Subgraphs contained in $c$-cyclic graphs with $c \geq 1$ and $\Delta=n-2$.

By computer calculations, we have $q_{2}\left(H_{13}\right) \doteq 3.3441, q_{2}\left(H_{14}\right) \doteq 2.7636$ and $q_{2}\left(H_{15}\right) \doteq 3.1404$.

\section{The maximum $Q$-separator of unicyclic graphs.}

Lemma 3.1. Let $U$ be a unicyclic graph with $n$ vertices. If $n \geq 7$ and $\Delta=n-3$, then $S_{Q}(U)<n-3$.

Proof. Let $U$ be a unicyclic graph with $n(n \geq 7)$ vertices. By Lemma 2.3, $q_{1}(U) \leq n$ holds. By Lemma 2.6, $U$ contains one of $H_{i}(i=1,2, \ldots, 12)$ as a subgraph. Then $U$ has $H_{i}+N_{n-7}$ as a spanning subgraph, where $N_{n-7}$ is the null graph of order $n-7$. By Lemma 2.1 and Table 2.1, if $U$ contains $H_{i}(i=1,2, \ldots, 11)$, then $q_{2}(U) \geq q_{2}\left(H_{i}+N_{n-7}\right)=q_{2}\left(H_{i}\right)>3$. We have $S_{Q}(U)<n-3$.

Suppose $U$ contains $H_{12}$ as a subgraph. Note that $\Delta=n-3$. By Lemmas 2.1 and 2.2 , then $q_{1}(U) \leq n-3+\frac{2 n-(n-3)-1-1}{n-3}=n-2+\frac{4}{n-3}$ and $q_{2}(U) \geq q_{2}\left(H_{12}+N_{n-7}\right)=$ $q_{2}\left(H_{12}\right)$. Hence $S_{Q}(U) \leq n-2+\frac{4}{n-3}-2.7935<n-3$ holds for $n \geq 7$. $\square$

Lemma 3.2. Let $U$ be a unicyclic graph with $n$ vertices. If $n \geq 7$ and $\Delta=n-2$, then $S_{Q}(U)<n-3$. 
Proof. Suppose $n \geq 7$. By Lemma 2.3, then $q_{1}(U) \leq n$. By Lemma 2.6, $U$ must contain $H_{13}, H_{14}$, or $H_{15}$ as a subgraph and $H_{j}+N_{n-7}$ a spanning subgraph. If $U$ contains $H_{13}$ or $H_{15}$, then $q_{2}(U) \geq q_{2}\left(H_{j}+N_{n-7}\right)=q_{2}\left(H_{j}\right)>3$. Hence $S_{Q}(U)<n-3$.

Suppose $U$ contains $H_{14}$ as a subgraph. Note that $\Delta=n-2$. By Lemma 2.2, then $q_{1}(U) \leq n-2+\frac{2 n-(n-2)-1}{n-2}=n-1+\frac{3}{n-2}$. Hence $S_{Q}(U) \leq n-1+\frac{3}{n-2}-2.7636<n-3$ holds for $n \geq 7$.

Denote by $U_{n}^{1}$ the unicyclic graph obtained from $C_{3}$ by attaching $n-3$ pendant edges to a common vertex of $C_{3}$.

LEMMA 3.3. If $n \geq 7$, then $n-3<S_{Q}\left(U_{n}^{1}\right)=r_{1}-r_{2}<n$, where $r_{1}$ and $r_{2}$ are the first two largest solutions of the equation $x^{3}-(3+n) x^{2}+3 n x-4=0$.

Proof. Consider the characteristic polynomial of $Q\left(U_{1}\right)$ which is

$$
\Phi\left(Q\left(U_{n}^{1}\right) ; x\right)=(x-1)^{n-3}\left[x^{3}-(3+n) x^{2}+3 n x-4\right] .
$$

Suppose $f(x)=x^{3}-(3+n) x^{2}+3 n x-4$. Note that $n \geq 7$. By direct calculations, we have

$$
\begin{gathered}
f(n+1)=n^{2}-n-6>0, \quad f(n)=-4<0, \quad f(3)=-4<0, \\
f(1)=2 n-6>0 \text { and } f(0)=-4<0 .
\end{gathered}
$$

The roots of the equation $f(x)=0$ lie in $(0,1),(1,3)$ and $(n, n+1)$. Then $n<q_{1}\left(U_{n}^{1}\right)<n+1$ and $1<q_{2}\left(U_{n}^{1}\right)<3$. Hence $n-3<S_{Q}\left(U_{n}^{1}\right)=r_{1}-r_{2}<n$.

LEMma 3.4. If $3 \leq n \leq 6$, then $S_{Q}(U) \leq S_{Q}\left(U_{n}^{1}\right)=r_{1}-r_{2}<n$ for $n=$ $3,4,5,6$, respectively, where $r_{1}$ and $r_{2}$ are the first two largest solutions of the equation $x^{3}-(3+n) x^{2}+3 n x-4=0$.

Proof. The unique unicyclic graph is $C_{3} \cong U_{3}^{1}$ if $n=3$. For $n=4$, there are two unicyclic graphs and $S_{Q}(U) \leq S_{Q}\left(U_{4}^{1}\right) \doteq 4.5616-2$. For $n=5$, there are 5 unicyclic graphs and $S_{Q}(U) \leq S_{Q}\left(U_{5}^{1}\right) \doteq 5.3234-2.3579$.

By the table of graphs on six vertices [8] and direct calculations among the 13 unicyclic graphs, $S_{Q}(U) \leq S_{Q}\left(U_{6}^{1}\right) \doteq 6.2015-2.5451$ holds.

Combining Lemmas 2.5 and 3.1-3.4, we arrive at the main result of this section:

THEOREM 3.5. Let $U$ be a unicyclic graph with $n$ vertices. If $n \geq 3$, then $S_{Q}(U) \leq S_{Q}\left(U_{n}^{1}\right)=r_{1}-r_{2}<n-1$, where $r_{1}$ and $r_{2}$ are the first two largest solutions of the equation $x^{3}-(3+n) x^{2}+3 n x-4=0$, and equality holds if and only if $U \cong U_{n}^{1}$. 
4. The maximum $Q$-separator of bicyclic graphs. By Lemma 2.5, we only need to consider bicyclic graphs with $\Delta \geq n-3$.

LEMMA 4.1. Let $B$ be a bicyclic graph with $n$ vertices. If $n \geq 7$ and $\Delta=n-3$, then $S_{Q}(B)<n-3$.

Proof. Similar to Lemma 3.1, if $B$ contains $H_{i}(i=1,2, \ldots, 11)$ as a subgraph, then $S_{Q}(B)<n-3$.

Suppose $B$ contains $H_{12}$ as a subgraph. By Lemma 2.2, then $q_{1}(B) \leq n-3+$ $\frac{2(n+1)-(n-3)-1-1}{n-3}=n-2+\frac{6}{n-3}$. Hence $S_{Q}(B) \leq n-2+\frac{6}{n-3}-2.7935<n-3$ holds for $n \geq 7$.

LEMMA 4.2. Let $B$ be a bicyclic graph with $n$ vertices. If $n \geq 7$ and $\Delta=n-2$, then $S_{Q}(B) \leq n-3$.

Proof. Similar to Lemma 3.2, if $B$ contains $H_{13}$ or $H_{15}$, then $S_{Q}(B)<n-3$.

Suppose $B$ contains $H_{14}$ as a subgraph. Note that $\Delta=n-2$ and $B$ is a bicyclic graph. Then $B$ contains a subgraph obtained from $H_{14}$ by adding an edge. There are six such subgraphs and the minimal $q_{2}$-value is 3 . Hence $S_{Q}(B) \leq n-3$.

The bicyclic graphs with $n$ vertices and $\Delta=n-1$ are $B_{n}^{1}$ and $B_{n}^{2}$ (Figure 3 ).

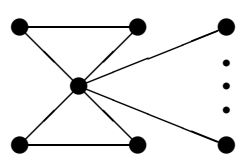

$B_{n}^{1}$

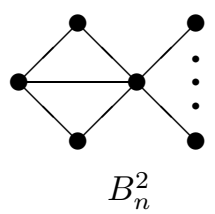

Figure 3. Bicyclic graphs with $\Delta=n-1$ and $n$ vertices.

In [7], by computer calculations, one of the present authors has obtained that the characteristic polynomials of $Q\left(B_{n}^{1}\right)$ and $Q\left(B_{n}^{2}\right)$ are $(x-1)^{n-4}(x-3)\left[x^{3}-(n+3) x^{2}+\right.$ $3 n x-8]$ and $(x-1)^{n-4}(x-2)\left[x^{3}-(n+4) x^{2}+4 n x-8\right]$, respectively.

LEMMA 4.3. If $n \geq 7$, then $S_{Q}\left(B_{n}^{2}\right)<n-3<S_{Q}\left(B_{n}^{1}\right)=r_{1}-3$, where $r_{1}$ is the largest solution of the equation $x^{3}-(n+3) x^{2}+3 n x-8=0$.

Proof. Let $f(x)=x^{3}-(n+3) x^{2}+3 n x-8$. We have

$$
\begin{gathered}
f(n+1)=n^{3}-n-10>0, \quad f(n)=-8<0, \quad f(3)=-8<0, \\
f(2)=2(n-6)>0 \text { and } f(0)=-8<0 .
\end{gathered}
$$

The roots of the equation $f(x)=0$ lie in $(0,2),(2,3)$ and $(n, n+1)$. Then 
$n<q_{1}\left(B_{n}^{1}\right)<n+1$ and $q_{2}\left(B_{n}^{1}\right)=3$. Hence $n-3<S_{Q}\left(B_{n}^{1}\right)=r_{1}-3$, where $r_{1}$ is the largest eigenvalue of the equation $x^{3}-(n+3) x^{2}+3 n x-8=0$.

Let $g(x)=x^{3}-(n+4) x^{2}+4 n x-8$. Note that $n \geq 7$. We have

$$
\begin{gathered}
g\left(n+\frac{1}{3}\right)=\frac{1}{27}\left(9 n^{2}-30 n-227\right)>0, \quad g(n)=-8<0, \\
g(4)=-8<0, \quad g\left(\frac{10}{3}\right)=\frac{4}{27}(15 n-104)>0, \\
g(1)=3 n-11>0 \text { and } g(0)=-8<0 .
\end{gathered}
$$

The roots of the equation $g(x)=0$ lie in $(0,1),\left(\frac{10}{3}, 4\right)$ and $\left(n, n+\frac{1}{3}\right)$. Then $n<q_{1}\left(B_{n}^{2}\right)<n+\frac{1}{3}$ and $\frac{10}{3}<q_{2}\left(B_{n}^{1}\right)<4$. Hence $S_{Q}\left(B_{n}^{2}\right)<n+\frac{1}{3}-\frac{10}{3}=n-3$.

LEMmA 4.4. Let $B$ ba a bicyclic graph with $n$ vertices. If $n=4,5$, or 6 , then $S_{Q}(B) \leq S_{Q}\left(B_{n}^{2}\right)$.

Proof. There is only one bicyclic graph $B_{4}^{2}$ for $n=4$. For $n=5$, there are five bicyclic graphs. By direct calculations, we have $S_{Q}(B) \leq S_{Q}\left(B_{5}^{2}\right) \doteq 5.7785-2.7108=$ 3.0677 .

By the table of graphs on six vertices [8], there are 19 bicyclic graphs. By direct calculations, then $S_{Q}(B) \leq S_{Q}\left(B_{6}^{2}\right) \doteq 6.4940-3.1099=3.3841$.

By Lemmas 2.5 and 4.1-4.4, we have:

THEOREM 4.5. Let $B$ be a bicyclic graph with $n$ vertices.

(1) If $n \geq 7$, then $S_{Q}(B) \leq S_{Q}\left(B_{n}^{1}\right)=r_{1}-3$, where $r_{1}$ is the largest solution of the equation $x^{3}-(n+3) x^{2}+3 n x-8=0$, and equality holds if and only if $U \cong B_{n}^{1}$.

(2) If $n=4,5$, or 6 , then $S_{Q}(B) \leq S_{Q}\left(B_{n}^{2}\right)$.

\section{The maximum $Q$-separator of tricyclic graphs.}

LEMMA 5.1. Let $F$ be a tricyclic graph with $n$ vertices. If $n \geq 9$ and $\Delta=n-3$, then $S_{Q}(F)<n-3$.

Proof. As in Lemmas 3.1 and 4.1, if $F$ contains $H_{i}(i=1,2, \ldots, 11)$ as a subgraph, then $S_{Q}(F)<n-3$.

Suppose $F$ contains $H_{12}$ as a subgraph. By Lemma 2.2, then $q_{1}(F) \leq n-3+$ $\frac{2(n+2)-(n-3)-1-1}{n-3}=n-2+\frac{8}{n-3}$. Hence $S_{Q}(F) \leq n-2+\frac{8}{n-3}-2.7935<n-3$ holds for $n \geq 9$. 
By the proof of Lemma 4.2, we similarly obtain:

LEMMA 5.2. Let $F$ be a tricyclic graph with $n$ vertices. If $n \geq 9$ and $\Delta=n-2$, then $S_{Q}(F) \leq n-3$.

Let $F$ be a tricyclic graph with $\Delta=n-1$. Then $F \cong F_{n}^{i}(i=1,2, \ldots, 5)$ depicted in Figure 4.

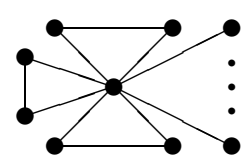

$F_{n}^{1}$

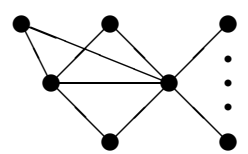

$F_{n}^{2}$

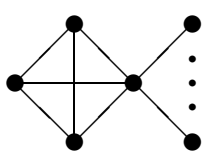

$F_{n}^{3}$

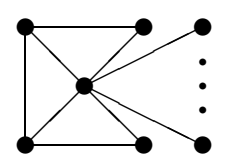

$F_{n}^{4}$

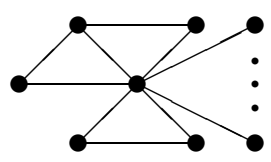

$F_{n}^{5}$

Figure 4. Tricyclic graphs with $\Delta=n-1$ and $n$ vertices.

LEMmA 5.3. If $n \geq 9$, then $S_{Q}\left(F_{n}^{1}\right)=r_{1}-3>n-3>S_{Q}\left(F_{n}^{i}\right)(i=2, \ldots, 5)$, where $r_{1}$ is the largest solution of the equation $x^{3}-(n+3) x^{2}+3 n x-12=0$.

Proof. By computer calculations or [7], the $Q$-characteristic polynomials of $F_{n}^{i}$ are as follows.

The characteristic polynomial of $Q\left(F_{n}^{1}\right)$ is

$$
\Phi\left(Q\left(F_{n}^{1}\right) ; x\right)=(x-1)^{n-5}(x-3)^{2}\left[x^{3}-(n+3) x^{2}+3 n x-12\right] .
$$

Let $f_{1}(x)=x^{3}-(n+3) x^{2}+3 n x-12$. Note that $n \geq 9$. Then

$$
\begin{gathered}
f_{1}(n+1)=n^{2}-n-14>0, \quad f_{1}(n)=-12<0, \quad f_{1}(3)=-12<0, \\
f_{1}(2)=2(n-8)>0 \text { and } f_{1}(0)=-12<0 .
\end{gathered}
$$

The roots of the equation $f_{1}(x)=0$ lie in $(0,2),(2,3)$ and $(n, n+1)$. Then $n<q_{1}\left(F_{n}^{1}\right)<n+1$ and $q_{2}\left(F_{n}^{1}\right)=3$. Hence $n-3<S_{Q}\left(F_{n}^{1}\right)$.

The characteristic polynomials of $Q\left(F_{n}^{2}\right)$ and $Q\left(F_{n}^{3}\right)$ are equal,

$$
\Phi\left(Q\left(F_{n}^{2}\right) ; x\right)=\Phi\left(Q\left(F_{n}^{3}\right) ; x\right)=(x-1)^{n-5}(x-2)^{2}\left[x^{3}-(n+5) x^{2}+5 n x-12\right] .
$$


Let $f_{2}(x)=f_{3}(x)=x^{3}-(n+5) x^{2}+5 n x-12$. Note that $n \geq 9$. Then

$$
\begin{gathered}
f_{2}\left(n+\frac{1}{3}\right)=\frac{1}{27}\left(9 n^{2}-39 n-338\right)>0, \quad f_{2}(n)=-12<0, \quad f_{2}(5)=-12<0 \\
f_{2}\left(\frac{10}{3}\right)=\frac{2}{27}(75 n-412)>0 \text { and } f_{2}(0)=-12<0 .
\end{gathered}
$$

The roots of the equation $f_{2}(x)=0$ lie in $\left(0, \frac{10}{3}\right),\left(\frac{10}{3}, 5\right)$ and $\left(n, n+\frac{1}{3}\right)$. Then $n<q_{1}\left(F_{n}^{2}\right)=q_{1}\left(F_{n}^{3}\right)<n+\frac{1}{3}$ and $\frac{10}{3}<q_{2}\left(F_{n}^{1}\right)=q_{1}\left(F_{n}^{3}\right)<5$. Hence $S_{Q}\left(F_{n}^{2}\right)=$ $S_{Q}\left(F_{n}^{3}\right)<n+\frac{1}{3}-\frac{10}{3}=n-3$.

The characteristic polynomial of $Q\left(F_{n}^{4}\right)$ is

$$
\Phi\left(Q\left(F_{n}^{4}\right) ; x\right)=(x-1)^{n-5}(x-3)\left[x^{4}-(n+6) x^{3}+(6 n+7) x^{2}-(7 n+12) x+20\right] .
$$

Let $f_{4}(x)=x^{4}-(n+6) x^{3}+(6 n+7) x^{2}-(7 n+12) x+20$. Note that $n \geq 9$. Then

$$
\begin{gathered}
f_{4}\left(n+\frac{1}{2}\right)=\frac{1}{16}\left(8 n^{3}-36 n^{2}-178 n+241\right)>0, \quad f_{4}(n)=-12 n+20<0, \\
f_{4}(5)=-10(n-1)<0, \quad f_{4}\left(\frac{7}{2}\right)=\frac{1}{16}(98 n-695)>0, \\
f_{4}(1)=-2 n+10<0 \text { and } f_{4}(0)=20>0 .
\end{gathered}
$$

The roots of the equation $f_{4}(x)=0$ lie in $(0,1),\left(1, \frac{7}{2}\right),\left(\frac{7}{2}, 5\right)$ and $\left(n, n+\frac{1}{2}\right)$. Then $n<q_{1}\left(F_{n}^{4}\right)<n+\frac{1}{2}$ and $\frac{7}{2}<q_{2}\left(F_{n}^{4}\right)<5$. Hence $S_{Q}\left(F_{n}^{4}\right)<n+\frac{1}{2}-\frac{7}{2}=n-3$.

The characteristic polynomial of $Q\left(F_{n}^{5}\right)$ is $\Phi\left(Q\left(F_{n}^{5}\right) ; x\right)=(x-1)^{n-5}(x-2)\left[x^{4}-(n+7) x^{3}+(7 n+12) x^{2}-(12 n+12) x+40\right]$. Let $f_{5}(x)=x^{4}-(n+7) x^{3}+(7 n+12) x^{2}-(12 n+12) x+40$. Note that $n \geq 9$. Then

$$
\begin{gathered}
f_{5}\left(n+\frac{1}{2}\right)=\frac{1}{16}\left(8 n^{3}-44 n^{2}-146 n+579\right)>0, \quad f_{5}(n)=-12 n+40<0, \\
f_{5}(4)=-8<0, \quad f_{5}\left(\frac{7}{2}\right)=\frac{1}{16}(14 n-81)>0, \\
f_{5}(2)=-4(n-6)<0 \text { and } f_{5}(0)=40>0 .
\end{gathered}
$$


The roots of the equation $f_{5}(x)=0$ lie in $(0,2),\left(2, \frac{7}{2}\right),\left(\frac{7}{2}, 4\right)$ and $\left(n, n+\frac{1}{2}\right)$. Then $n<q_{1}\left(F_{n}^{5}\right)<n+\frac{1}{2}$ and $\frac{7}{2}<q_{2}\left(F_{n}^{5}\right)<4$. Hence $S_{Q}\left(F_{n}^{5}\right)<n+\frac{1}{2}-\frac{7}{2}=n-3$.

By Lemmas 2.5 and 5.1-5.3, we have:

THEOREM 5.4. Let $F$ be a tricyclic graph with $n$ vertices. If $n \geq 9$, then $S_{Q}(F) \leq$ $S_{Q}\left(F_{n}^{1}\right)=r_{1}-3$, where $r_{1}$ is the largest solution of the equation $x^{3}-(n+3) x^{2}+$ $3 n x-12=0$, and equality holds if and only if $U \cong F_{n}^{1}$.

Acknowledgement. The authors are very grateful to the anonymous referees for their valuable comments and suggestions, which have improved the presentation of the paper.

\section{REFERENCES}

[1] J.X. Li, W.C. Shiu, and W.H. Chan. Some results on the Laplacian eigenvalues of unicyclic graphs. Linear Algebra Appl., 430:2080-2093, 2008.

[2] K.C. Das. On conjectures involving second largest signless Laplacian eigenvalue of graphs. Linear Algebra Appl., 432:3018-3029, 2010.

[3] D. Cvetković, P. Rowlinson, and S.K. Simić. Eigenvalue bounds for the signless Laplacian. Publ. Inst. Math. (Beograd), 81(95):11-27, 2007.

[4] R. Merris. Laplacian matrices of graphs: a survey. Linear Algebra Appl., 197/198:143-176, 1994.

[5] D. Cvetković and S.K. Simić. Towards a spectral theory of graphs based on signless Laplacian I. Publ. Inst. Math. (Beograd), 85(99):19-33, 2009.

[6] K.C. Das. The Laplacian spectrum of a graph. Comput. Math. Appl., 48:715-724, 2004.

[7] M. Liu and B. Liu. On the signless Laplacian spectra of bicyclic and tricyclic graphs. ARS Combin., to appear.

[8] D. Cvetković and M. Petrić. A table of connected graphs on six vertices. Disc. Math., 50:37-49, 1984. 